\title{
Crowdfunding para el rescate de microempresas. Factores y percepciones de inversionistas potenciales en México
}

\section{Crowdfunding for the rescue of micro-businesses. Factors and perceptions of potential investors in Mexico}

Dr. Francisco Javier Segura-Mojica es profesor investigador del Tecnológico Nacional de México/Instituto Tecnológico de San Luis Potosí (recursosmx@yahoo.com) (orcid.org/0000-0001-5981-9246)

\begin{abstract}
Resumen
Durante el confinamiento asociado a la contingencia sanitaria por la COVID-19, casi cuatrocientas mil micro y pequeñas empresas se vieron forzadas a cerrar en México. En este documento se presentan los resultados de una investigación cuantitativa, de alcance descriptivo, cuyo objetivo fue rastrear percepciones de microinversionistas y factores que influirían sobre su decisión de ayudar al rescate de microempresas y empleos en el escenario de la contingencia económica derivada de la COVID-19. Como metodología se utilizó la Actor-Network Theory (ANT) y como herramienta de análisis el Chi Square Automatic Interaction Detection (CHAID). Se encontró que, en el escenario de recesión económica, aproximadamente $23 \%$ de las personas económicamente activas cuentan con excedentes que podrían destinarse al financiamiento colectivo; además, se ha reforzado el interés de los micro inversionistas hacia proyectos que crean empleos o ayudan a conservar los ya existentes; por otra parte, como elemento que ayuda a rastrear las asociaciones entre inversionistas y emprendedores se identificó una variable que llamamos mediación; con base en lo anterior, se definió un listado de criterios que podrían ayudar a diseñar una plataforma de financiamiento colectivo con enfoque de red descentralizada y solidaria. Además, se encontró que la digitalización es clave para que una microempresa aspire a obtener financiamiento colectivo, y por lo tanto, para mejorar sus posibilidades de sobrevivencia en un escenario de contingencia económica.
\end{abstract}

\begin{abstract}
During the confinement associated with the health contingency due to Covid-19, almost four hundred thousand micro and small businesses were forced to close in Mexico. This document presents the results of a quantitative descriptive and cross-sectional study, the objective of which was to track the perceptions of micro-investors and factors that would influence their decision to help rescue micro-enterprises and jobs in the scenario of the economic contingency. The Actor-Network Theory (ANT) was used as the analysis methodology. It was found that, in the scenario of economic recession associated with the health contingency, approximately $23 \%$ of economically active people have surpluses that could be used for collective financing; Furthermore, the interest of micro-investors in projects that create jobs or help to preserve existing ones has been reinforced. On the other hand, as an element that helps to trace the associations between investors and entrepreneurs, a variable that we call mediation was identified; Based on the above, a list of criteria was defined that could help design a collective financing platform with a decentralized and solidarity network approach. In addition, it was found that digitization is key for a microenterprise to aspire to obtain collective financing, and therefore, to improve its chances of survival in a scenario of economic contingency.
\end{abstract}

\section{Palabras clave I keywords}

Microempresa, crowdfunding, actor-red, microinversionistas, emprendedores, COVID-19, fintech, plataforma. Micro-enterprise, crowdfunding, actor-network, micro-investors, entrepreneurs, COVID-19, fintech, platform.

Cómo citar: Segura-Mojica, F.J. (2021). Crowdfunding para el rescate de microempresas. Factores y percepciones de inversionistas potenciales en México. Retos Revista de Ciencias de la Administración y Economía, 11(21), pp. 71-91. https://doi.org/10.17163/ret.n21.2021.05 


\section{Introducción}

Como resultado de la ralentización de la economía global, y en específico, de la economía mexicana en el escenario de la contingencia por COVID-19, miles de micro y pequeñas empresas han cerrado o se encuentran en riesgo de quiebra, y con ello, es posible la desaparición de millones de empleos, dada la imposibilidad de un sector de las microempresas para solventar sus gastos operativos. El Estudio sobre Demografía de los Negocios (EDN) 2020, del Instituto Nacional de Estadística y Geografía (INEGI, 2020), muestra que, entre mayo de 2019 y septiembre de 2020, el número de micro, pequeñas y medianas empresas en México se redujo de 4,85 a 4,46 millones, lo que representa una disminución de $8,06 \%$ en el número de establecimientos.

Según estimaciones del Banco Interamericano de Desarrollo (BID, 2020), México podría perder entre el $4,1 \%$ de los empleos formales en caso de una crisis de corto plazo, hasta el 14,4\% en caso de una recesión prolongada.

De acuerdo con los Censos Económicos 2019, el 95\% de los establecimientos formales cuentan con menos de diez trabajadores (INEGI, 2019). Estos negocios son altamente vulnerables ante situaciones de contingencia, pues muchos de ellos no cuentan con reservas económicas para sobrevivir a recesos prolongados como el ocasionado por la contingencia sanitaria.

\subsection{Crowdfunding o financiamiento colectivo}

Las crisis sanitaria y económica asociadas a la COVID-19, han puesto en evidencia las debilidades del modelo capitalista y de la democracia liberal. Ante la velocidad del contagio, se revelaron fuertes limitaciones tanto del Estado como de las instituciones financieras y del sector privado en la implementación de acciones eficaces para la contención del brote epidémico, la preservación del sistema productivo, y la protección de los estratos sociales especialmente vulnerables. Esto se asocia con lo global e interdependiente del sistema productivo, pero también con lo asimétrico y excluyente de los sistemas financieros. Sin embargo, la incertidumbre generada por la COVID-19 también nos ofrece pistas sobre la manera en que elementos asociados a la sociedad del conocimiento y a la economía solidaria, pueden ayudar a construir soluciones que podríamos llamar "pos-capitalistas" (Nelson, 2020).

El crowdfunding (CF) es un fenómeno en el que concurren factores propios de la sociedad de la información y el conocimiento: digitalización, nuevos espacios de convivencia con una mediación tecnológica, y modelos alternativos de emprendimiento y financiación. García y Garibay (2016), definen CF como "un método a través del cual individuos u organizaciones sin fines de lucro y empresas obtienen recursos para realizar iniciativas o proyectos de carácter social o empresarial" (p. 37). Otros autores, como Schwienbacher y Larralde (2010), y Belleflame et al. (2012), coinciden al conceptualizar el CF como una llamada que utiliza el internet para proveer de recursos financieros a un proyecto. Estos fondos pueden tomar la forma de donaciones, préstamos u otorgarse a cambio de algún tipo de recompensa.

La modalidad de financiamiento colectivo utilizando el internet se observó a partir de 1997 cuando grupos musicales comenzaron a recaudar donaciones para financiar giras (García \& Garibay, 2016). Los mismos autores ubican a la empresa Artist Share, en el año 2003, como la primera plataforma de financiamiento colectivo enfocada al sector artístico, y a la empresa Kickstarter en 2008, como la primera enfocada a los negocios.

De acuerdo con Salido et al. (2019), la mayoría de los modelos de CF están basados en el concepto de equity o inversión de capital, es decir, una recompensa económica que puede significar participación en la propiedad del proyecto. Sin embargo, existe un creciente interés en algunos segmentos de la población por apoyar causas sin fines de lucro. 
El CF ha sido examinado desde la perspectiva económica como una oportunidad para captar excedentes económicos de los consumidores y limitar los precios en la oferta de bienes y servicios para atraer un mayor número de proveedores de fondos (Belleflame et al., 2012; Bahena et al. 2017). Por otra parte, autores como Agrawal et al. (2011) destacan el rol que juega el CF para acortar las distancias entre inversionistas y emprendedores, reduciendo efectos no deseados del financiamiento bancario.

Salvador (2018) apunta que la falta de liquidez del sistema bancario es un factor que impulsa la operación de plataformas de $\mathrm{CF}$, y que esto se ha dado en especial a partir de la crisis financiera de los años 2008 y 2009, por lo que actualmente se vive una transición del modelo tradicional de capital de riesgo a plataformas de financiación participativa en el que el poder de la multitud ayuda a satisfacer las demandas de crédito.

\subsection{Ecosistema de Tecnología Financiera (Fintech)}

Además de las restricciones y dificultades que se han derivado del confinamiento por la COVID-19, en México existe una problemática estructural de inclusión financiera y acceso al crédito. De acuerdo con Demirguc-Kunt et al. (2015), en México solo el $39 \%$ de la población adulta tiene una cuenta bancaria en una institución formal.

León y Saavedra (2018) advierten que, para las Mipymes, el financiamiento bancario además de costoso, es asimétrico; las microempresas son vistas por los bancos como clientes de riesgo ante la carencia de garantías hipotecarias, alta mortalidad, baja productividad, y la posibilidad de que cambien el destino de los recursos prestados. La tasa de interés se determina por selección adversa al ser difícil distinguir entre pagadores y no pagadores, y ello, aunado a las imperfecciones del mercado de capitales, se convierte en un problema de racionamiento que afecta en especial a las empresas de menor tamaño. De acuerdo con el Banco de México (2015), en México, solo el 29,5\% de las empresas con menos de 100 empleados cuenta con financiamiento bancario.

Este conjunto de variables ha configurado un escenario propicio para el surgimiento de actores emergentes que ayudan a resolver las asimetrías del mercado de financiamiento, como las empresas de tecnología financiera (Fintech). Las empresas e iniciativas Fintech forman parte de un ecosistema que, utilizando la telefonía móvil y la innovación, amplían la cobertura de los servicios financieros con menores costos, información mejor distribuida, mayor transparencia y accesibilidad (Zedeli, 2019).

En un estudio realizado en micro y pequeñas empresas de 22 países afiliados a la Organización para la Cooperación y el Desarrollo Económico (OCDE), Abbasi et al. (2020) encontraron una relación positiva entre la eficiencia de las Mipymes y el uso de las Fintech, por lo que sugieren a los gobiernos introducir políticas de apoyo a este tipo de iniciativas. Por otra parte, al analizar factores de éxito en el financiamiento de Mipymes a través de CF, González et al. (2021) encuentran que el riesgo es la variable de mayor impacto sobre el fondeo exitoso.

En México se ha desarrollado en años recientes un esquema regulatorio que favorece el crecimiento del ecosistema Fintech. De acuerdo con Oxford Analítica (2019), el país es ahora líder en tecnología financiera a nivel Latinoamérica. Este marco regulatorio tiene como pieza clave la Ley para Regular las Instituciones de Tecnología Financiera, también conocida como Ley Fintech. En ella se incluye a las entidades de financiamiento colectivo y fondos de pago electrónico como Instituciones de Tecnología Financiera (ITF). El Financiamiento Colectivo es definido en la Ley Fintech como "las actividades destinadas a poner en contacto a personas del público en general, con el fin de que entre ellas se otorguen financiamientos" (Secretaría de Hacienda y Crédito Público, 2018, p. 6). 
González (2018) considera que la Ley Fintech mexicana es una ventana de oportunidad que permite la entrada de nuevos actores al escenario del financiamiento empresarial en igualdad de condiciones con los bancos; al abrir la competencia, se hace posible una oferta de más y mejores servicios financieros en menos tiempo y a menores precios, además de que motiva a los emprendedores a avanzar en sus proyectos accediendo a nuevas fuentes de capital.

\subsection{De las empresas Fintech a la teoría del actor-red}

Una manera de aproximarse a la forma en que se integran las redes de financiamiento colectivo es lo que se conoce como Actor Network Theory (ANT) que Law (2009) define como "una familia diversa de herramientas semiótico-materiales, sensibilidades y métodos de análisis que tratan todos los elementos de los mundos naturales y sociales como un efecto, generado permanentemente, de las redes de relaciones dentro de las que están ubicados" (p.141).

Latour (2008) destaca que lo social se construye con la participación de personas, organizaciones y entidades no sociales, como la tecnología, y discurre a través asociaciones y movimientos (proceso continuo de reensamblajes) que si se rastrean sistemáticamente terminan en una definición compartida de mundo, o "colectivo".

Para integrar lo colectivo, se lleva a cabo un proceso llamado traducción (Jarrahi \& Sawyer, 2019), que es la secuencia de un grupo de actores que intentan avanzar sobre una agenda, y para ello movilizan sus recursos y traducen los intereses de los demás con la esperanza de involucrarlos, transformando cuestiones de hecho por cuestiones de interés (García, 2017). Según Iyamu et al. (2018), existen cuatro momentos en este proceso: problematización, interés, incorporación y movilización (Figura 1).

\section{Figura 1. Proceso de traducción en la Actor Network Theory}

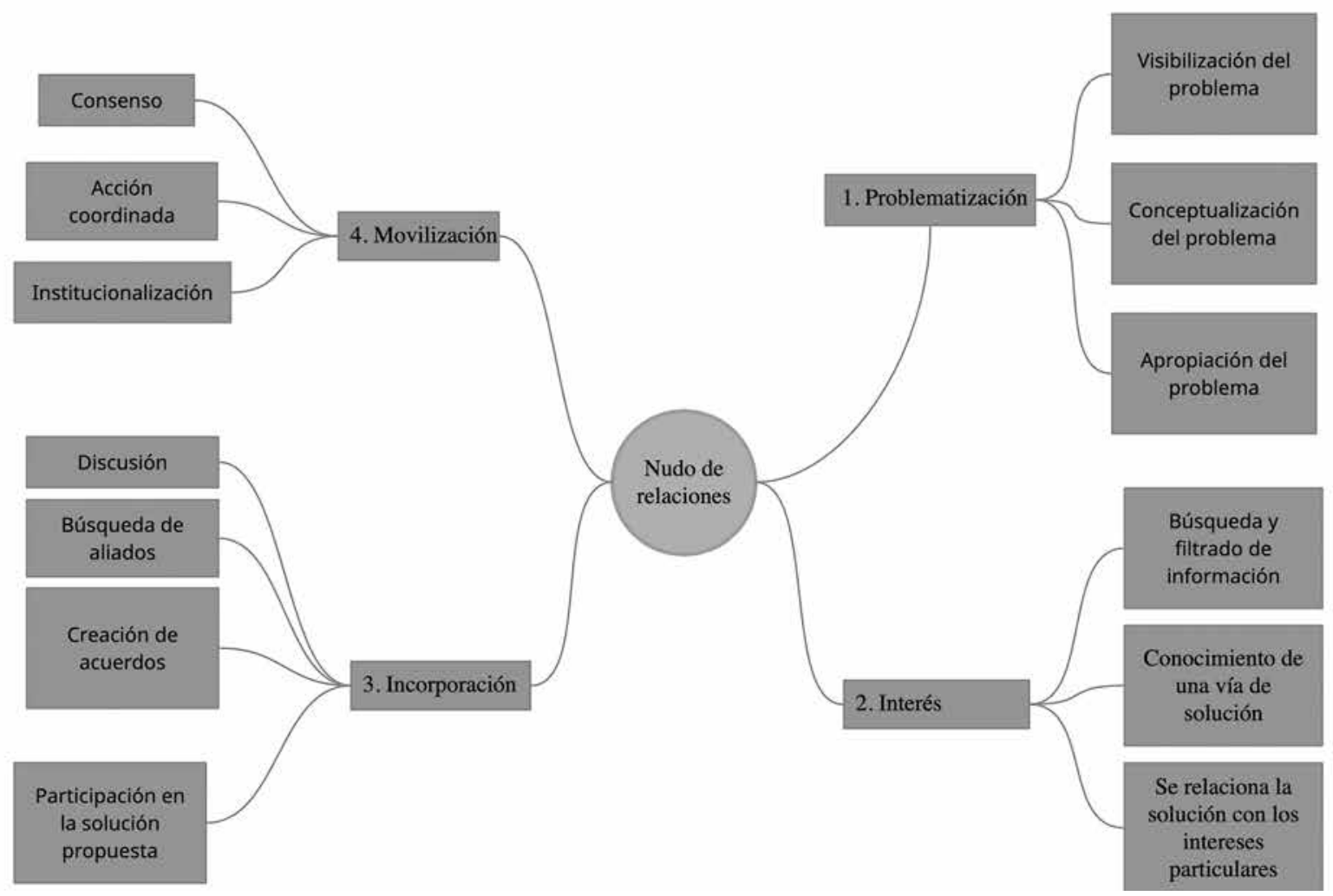

Fuente: Elaboración propia a partir de Iyamu et al. (2018); Sánchez et al. (2020). 
El proceso de traducción es relevante para nuestro análisis debido a que es un modelo que ayuda a interpretar las redefiniciones y cambios de intereses de los actores. De acuerdo con Ruiz (1999) implica modificaciones lingüísticas y geométricas. En el escenario de la contingencia por la COVID-19, podemos observar una traducción en el sentido lingüístico conforme los actores comienzan a reinterpretar y a verbalizar su realidad a partir de los cambios, reales e imaginarios que se han producido durante el confinamiento; y en sentido geométrico, en la medida en que participan en nuevas asociaciones y comienzan a jugar roles diferentes a los que desempeñaban.

\section{Materiales y métodos}

Las preguntas que dan origen a la presente investigación son: ¿Se pueden rastrear asociaciones entre actores del ecosistema de financiamiento? Y a partir de esas asociaciones ¿cuáles son los criterios que una microempresa o un proyecto de emprendimiento debería cumplir para atraer el interés de los microinversionistas?

Se realizó una investigación de tipo cuantitativo, alcance descriptivo, diseño transversal no experimental. Como metodología se utilizó la teoría del actor-red (ANT). De acuerdo con Echeverría y González (2009), ANT es una teoría descriptiva que permite caracterizar al mundo natural y social como producto de las redes de relaciones entre actores, que pueden ser tanto humanos como no humanos, y que según McBride (2003), provee una descripción social y técnica de los procesos de organización y asociación entre elementos heterogéneos.

Para recabar la información se diseñó un cuestionario que consta de cuatro preguntas de identificación sociodemográfica (edad, género, estado civil, lugar de residencia y nivel de estudios); y 14 preguntas relacionadas con las variables predictoras y criterio de la investigación (Tablas 1 y 4). El instrumento de recolección de información se aplicó a través de la plataforma Microsoft Forms durante los meses de agosto, septiembre y octubre de 2020.

Se empleó un muestreo por redes sociales (Heckathorn, 1997), que combina la técnica de bola de nieve con un sistema de selección controlada, de manera que los sujetos iniciales son los reclutadores (proponen a otros sujetos que tienen alguna característica semejante) y cada participante puede actuar como reclutador hasta que se logra el tamaño de muestra deseable (Baltar \& Gorjup, 2011). Este tipo de muestreo es utilizado en poblaciones ocultas, las cuales se caracterizan por su dificultad de identificación, falta de un marco muestral y distribución geográfica poco conocida (Marpsata \& Razafindratsimab, 2010). En este caso, la población objeto de estudio, definida como "microinversionistas potenciales" reúne características de población oculta. Utilizando un nivel de confianza de $94 \%$ y un margen de error de $6 \%$, se obtuvo un tamaño de muestra de 245 personas, de las cuáles 242 proporcionaron información válida.

\section{Tabla 1. Modelo teórico de la investigación. Variables predictoras y variable criterio}

\begin{tabular}{|c|c|}
\hline Variables predictoras & Variable criterio \\
\hline Edad (E) & \multirow{5}{*}{$\begin{array}{l}\text { Disponibilidad para realizar aportaciones } \\
\text { vía CF (DACF). Categorías: } 0=\mathrm{No}, 1=\mathrm{Mi}- \\
\text { croaportaciones, } 2=\text { Aportaciones de capi- } \\
\text { tal }\end{array}$} \\
\hline Género (G) & \\
\hline Estado civil (EC) & \\
\hline Escolaridad (ESC) & \\
\hline $\begin{array}{l}\text { Percepción de cambios en la situación económica } \\
\text { asociados al Covid-19 (PCE) }\end{array}$ & \\
\hline
\end{tabular}




\section{Variables predictoras}

Grado de afectación económica asociada al Covid-19 (GAE)

Nivel de solvencia económica durante la pandemia (SE)

Estabilidad en el empleo (EE)

Grado de involucramiento en campañas de CF (GICF)

Enfoque altruismo vs provecho personal (EAP)

Enfoque local vs global (LG)

Grado de esfuerzo para conseguir información (EI)

Tipo de beneficio esperado (BE)

Factores que hacen atractivo un proyecto (FA)

Recursos tecnológicos asociados a la mediación (RM)

Factores que generan confianza (FC)

Factores que generan desconfianza (FD)

Fuente: Elaboración propia.

Para validar el cuestionario, se realizó un Análisis Factorial Exploratorio (AFE) utilizando el método de Análisis de Componentes Principales y Rotación Varimax, en el cual se determinó que existen cuatro factores que explican el $45.3 \%$ de la varianza total (Tabla 2).

Tabla 2. Matriz rotada de componentes. Rotación Varimax

\begin{tabular}{|l|c|c|c|c|c|}
\hline Variable & Factor1 & Factor2 & Factor3 & Factor4 & Communality \\
\hline PCE & 0.699 & -0.155 & 0.004 & 0.064 & 0.517 \\
\hline GAE & 0.676 & -0.279 & 0.014 & 0.125 & 0.551 \\
\hline SE & 0.044 & -0.081 & -0.044 & 0.838 & 0.713 \\
\hline EE & 0.582 & -0.023 & -0.022 & -0.338 & 0.454 \\
\hline GICF & 0.206 & 0.138 & 0.731 & 0.074 & 0.602 \\
\hline TB & 0.444 & 0.056 & -0.349 & -0.005 & 0.322 \\
\hline LG & -0.025 & 0.777 & -0.008 & -0.020 & 0.604 \\
\hline DACF & 0.596 & 0.442 & 0.155 & 0.030 & 0.575 \\
\hline EI & 0.180 & -0.038 & -0.327 & 0.203 & 0.182 \\
\hline RM & 0.127 & 0.399 & 0.038 & 0.450 & 0.380 \\
\hline FC & -0.149 & 0.630 & -0.094 & -0.041 & 0.430 \\
\hline FD & -0.016 & 0.129 & -0.723 & -0.052 & 0.543 \\
\hline EAP & -0.245 & 0.413 & 0.147 & 0.364 & 0.384 \\
\hline FA & -0.090 & -0.021 & 0.200 & 0.191 & 0.085 \\
\hline & & & & & \\
\hline Variance & 2.0215 & 1.6754 & 1.3849 & 1.2605 & 6.3423 \\
\hline \% Var & 0.144 & 0.120 & 0.099 & 0.090 & 0.453 \\
\hline
\end{tabular}

Fuente: Elaboración propia utilizando Minitab-19. 
Después de la rotación Varimax, la matriz de componentes muestra que el primer factor agrupa, con puntuaciones altas, los ítems relacionados con la percepción sobre las afectaciones generadas a partir del confinamiento por la COVID-19, mientras que el segundo factor agrupa elementos relacionados con la mediación, es decir, los intereses de los microinversionistas, los aspectos que les generan confianza y los recursos tecnológicos preferentes.

\section{Resultados}

\subsection{Descripción del ecosistema de financiamiento colectivo}

El primer paso del análisis consiste en dibujar la red inversionista-emprendedor. El objetivo es establecer un punto de partida para rastrear la conformación de asociaciones específicas. En la Figura 2 se muestran la generalidad de los elementos identificados entre los cuáles puede establecerse algún tipo de asociación, es decir, el "panorama", de acuerdo a la terminología de Latour (2008). Esto es lo que Correa-Moreira (2012) llama trama o actor-red.

\section{Figura 2. Ecosistema inversionista-emprendedor}

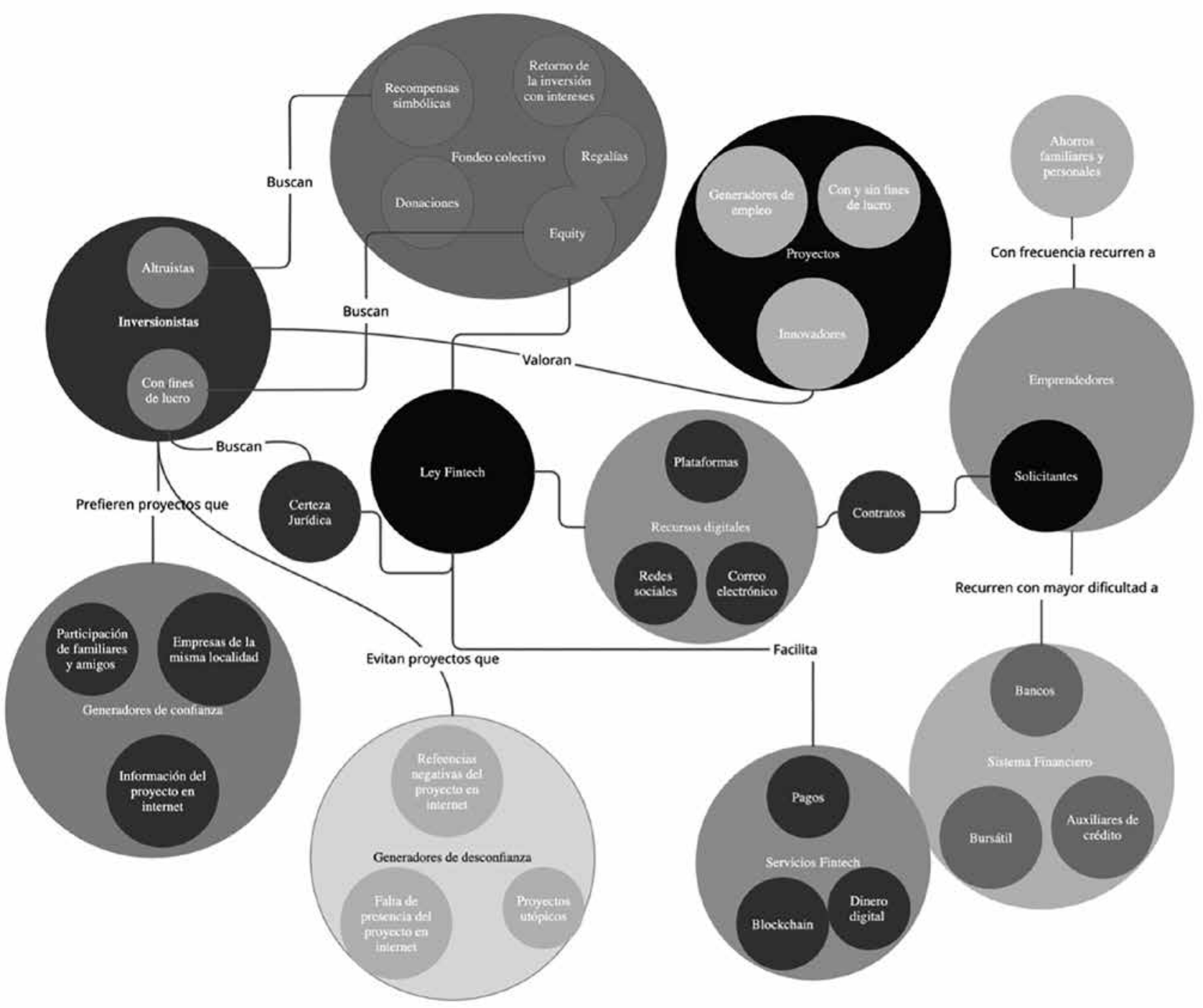

Fuente: Elaboración propia. 


\subsection{Análisis de respuestas y comportamientos asociados a las fases de traducción}

La segunda parte del ejercicio consistió en analizar la base de datos construida a partir de la encuesta de referencia para identificar lo que Latour (2008) llama cuestiones de interés.

En primer lugar, se utilizó estadística descriptiva (frecuencias y porcentajes), para caracterizar los resultados de la encuesta (Tablas 3 y 4). Para ello, se usó el software estadístico XLSTAT.

Tabla 3. Perfil sociodemográfico de la muestra

\begin{tabular}{|l|l|c|c|}
\hline \multicolumn{1}{|c|}{ Variable } & \multicolumn{1}{|c|}{ Categorías } & Frecuencias & $\%$ \\
\hline Edad & De 20 a 29 años & 133 & 55.649 \\
\hline & De 30 a 39 años & 17 & 7.113 \\
\hline & De 40 a 49 años & 32 & 13.389 \\
\hline & De 50 a 59 años & 29 & 12.134 \\
\hline & De 60 a 69 años & 9 & 3.766 \\
\hline Estado civil & Menos de 20 años & 19 & 7.950 \\
\hline & Casado & 10 & 22.594 \\
\hline \multirow{2}{*}{ dios? } & Otro & 175 & 73.222 \\
\hline & Soltero & 62 & 25.941 \\
\hline & Bachillerato o estudios técnicos & 130 & 54.393 \\
\hline & Licenciatura & 43 & 17.992 \\
\hline Sexo & Posgrado & 4 & 1.674 \\
\hline & Secundaria & 147 & 61.506 \\
\hline & Femenino & Masculino & 38.494 \\
\hline
\end{tabular}

Fuente: Elaboración propia. 
Tabla 4. Tabla de frecuencias por pregunta

\begin{tabular}{|c|c|c|c|c|c|c|c|c|c|}
\hline Variable & Ítem & $\mathbf{a}$ & b & c & d & $\mathbf{e}$ & $\mathbf{f}$ & $\mathbf{g}$ & h \\
\hline PCE & $\begin{array}{l}\text { Como resultado de la contingen- } \\
\text { cia sanitaria asociada al Covid-19 y } \\
\text { sus efectos en el sector productivo, } \\
\text { consideras que tu situación econó- } \\
\text { mica personal: a) Ha mejorado; b) } \\
\text { Ha empeorado; c) Se mantiene igual } \\
\text { que antes. }\end{array}$ & 8 & 148 & 86 & & & & & \\
\hline GAE & $\begin{array}{l}\text { ¿Cuáles de los siguientes efectos } \\
\text { asociados a la contingencia por } \\
\text { Covid-19 has sufrido?: a) Perdí mi } \\
\text { empleo; b) Algún familiar cercano } \\
\text { perdió su empleo; c) Algún conoci- } \\
\text { do perdió su empleo; d) Se reduje- } \\
\text { ron mis ingresos; e) Se redujeron los } \\
\text { ingresos de mi familia; f) Cerramos } \\
\text { algún negocio personal o familiar; g) } \\
\text { Tengo un negocio cuyos ingresos se } \\
\text { redujeron de manera significativa; h) } \\
\text { Ninguno de los anteriores. }\end{array}$ & 33 & 78 & 55 & 89 & 125 & 15 & 35 & 38 \\
\hline SE & $\begin{array}{l}\text { ¿Qué tan fácil o difícil es para ti } \\
\text { cubrir los gastos del mes?: a) Ter- } \\
\text { mino el mes sin problemas y tengo } \\
\text { un excedente que puedo invertir para } \\
\text { ahorrar o financiar proyectos; b) Jus- } \\
\text { to, pero cubro los gastos del mes; c) } \\
\text { A veces no me alcanza para llegar a } \\
\text { fin de mes; d) Normalmente no me } \\
\text { alcanza para llegar a fin de mes. }\end{array}$ & 55 & 108 & 59 & 20 & & & & \\
\hline EE & $\begin{array}{l}\text { Actualmente ¿cuentas con algún } \\
\text { trabajo remunerado, aunque sea } \\
\text { temporal?: a) Si, trabajo de manera } \\
\text { permanente para una empresa o ins- } \\
\text { titución; b) Si, cuento con mi propio } \\
\text { negocio; c) Si, trabajo de manera tem- } \\
\text { poral para una empresa o institución; } \\
\text { d) Si, realizo trabajos temporales por } \\
\text { mi propia cuenta; e) Me encuentro } \\
\text { desempleado. }\end{array}$ & 90 & 19 & 32 & 29 & 72 & & & \\
\hline GICF & $\begin{array}{l}\text { ¿Alguna vez has apoyado con dine- } \\
\text { ro una campaña de financiamiento } \\
\text { colectivo o crowdfunding?: a) Si; b) } \\
\text { No, aunque sí conozco el concepto de } \\
\text { crowdfunding o financiamiento co- } \\
\text { lectivo; c) No y tampoco estoy fami- } \\
\text { liarizado con el concepto de financia- } \\
\text { miento colectivo. }\end{array}$ & 15 & 50 & 177 & & & & & \\
\hline
\end{tabular}




\begin{tabular}{|c|c|c|c|c|c|c|c|c|c|}
\hline Variable & Ítem & a & b & c & d & e & f & $\mathrm{g}$ & h \\
\hline EAP & $\begin{array}{l}\text { ¿Cuáles de los siguientes factores } \\
\text { influirían para que tomaras la deci- } \\
\text { sión de apoyar un proyecto a través } \\
\text { de crowdfunding o financiamien- } \\
\text { to colectivo? a) Que el proyecto sea } \\
\text { creativo o innovador; b) Que el apoyo } \\
\text { permita salvar una o varias fuentes de } \\
\text { empleo; c) Que el apoyo permita crear } \\
\text { uno o varios nuevos empleos; d) Que } \\
\text { el apoyo me genere algún beneficio } \\
\text { simbólico e) Que el apoyo me gene- } \\
\text { re algún beneficio económico f) Que } \\
\text { sea altruista. Por ejemplo, que apoye } \\
\text { a personas en situación de pobreza; } \\
\text { g) Ninguno de los anteriores. No me } \\
\text { interesa apoyar proyectos por crowd- } \\
\text { funding. }\end{array}$ & 87 & 89 & 103 & 35 & 82 & 65 & 18 & \\
\hline LG & $\begin{array}{l}\text { Si te invitaran a participar en una } \\
\text { campaña de financiamiento colecti- } \\
\text { vo para rescatar microempresas en } \\
\text { riesgo de cierre por la contingen- } \\
\text { cia sanitaria derivada del Covid-19, } \\
\text { ¿Qué proyectos preferirías apoyar?: } \\
\text { a) Empresas de personas conocidas; b) } \\
\text { Empresas de mi localidad; c) Empre- } \\
\text { sas de cualquier localidad, pero que se } \\
\text { encuentren en México d) Empresas de } \\
\text { otros países; e) No me interesa partici- } \\
\text { par en este tipo de campañas. }\end{array}$ & 104 & 132 & 77 & 7 & 15 & & & \\
\hline DACF & $\begin{array}{l}\text { Si decidieras participar en una } \\
\text { campaña de financiamiento colec- } \\
\text { tivo para rescatar microempresas } \\
\text { en riesgo de cierre ¿Cuál sería el } \\
\text { monto de tu aportación?: a) Menos } \\
\text { de } 100 \text { pesos; b) De } 101 \text { a } 500 \text { pesos; } \\
\text { c) De } 501 \text { a } 1000 \text { pesos; d) De } 1000 \text { a } \\
5000 \text { pesos; e) Más de } 5000 \text { pesos; f) } \\
\text { No puedo aportar recursos; g) No me } \\
\text { interesa aportar recursos }\end{array}$ & 39 & 76 & 40 & 41 & 5 & 32 & 9 & \\
\hline EI & $\begin{array}{l}\text { ¿Qué información de un proyecto } \\
\text { investigarías para estar dispues- } \\
\text { to a apoyarlo con micro financia- } \\
\text { miento?: a) Descripción general de } \\
\text { la empresa, su mercado y sus produc- } \\
\text { tos; b) Perfil de los emprendedores } \\
\text { o responsables de proyecto c) Situa- } \\
\text { ción fiscal y financiera del negocio; d) } \\
\text { Historia del proyecto e) Otra }\end{array}$ & 180 & 112 & 107 & 64 & 14 & & & \\
\hline
\end{tabular}




\begin{tabular}{|c|c|c|c|c|c|c|c|c|c|}
\hline Variable & Ítem & a & b & c & d & e & f & $\mathrm{g}$ & h \\
\hline $\mathbf{B E}$ & $\begin{array}{l}\text { Si apoyaras algún proyecto de em- } \\
\text { prendimiento o microempresa a } \\
\text { través de una campaña de finan- } \\
\text { ciamiento colectivo ¿Qué espera- } \\
\text { rías recibir?: a) Nada en específico; } \\
\text { b)Información detallada del uso que } \\
\text { se dio a los recursos c) Que las apor- } \\
\text { taciones sean deducibles de impues- } \\
\text { tos; d) Que las aportaciones me sean } \\
\text { devueltas como si fuera un présta- } \\
\text { mo; e) Que las aportaciones me sean } \\
\text { devueltas con un interés; f) Que las } \\
\text { aportaciones me permitan convertir- } \\
\text { me en socio del negocio; g) Que los } \\
\text { emprendedores me envín alguna } \\
\text { recompensa simbólica (por ejemplo } \\
\text { un souvenir o una carta de agradeci- } \\
\text { miento) }\end{array}$ & 34 & 117 & 49 & 40 & 38 & 83 & 33 & \\
\hline FA & $\begin{array}{l}\text { ¿Cuál de los siguientes factores } \\
\text { influiría para hacer atractivo un } \\
\text { proyecto con fines de micro finan- } \\
\text { ciamiento?: a) Que me inspire con- } \\
\text { fianza; b) Que sea factible, no muy } \\
\text { utópico c) Que esté contado en for- } \\
\text { ma atractiva; d) Que proponga hacer } \\
\text { cosas de alta calidad; e) Que plantee } \\
\text { alguna innovación o desarrollo cien- } \\
\text { tífico y tecnológico; f) Que genere em- } \\
\text { pleos y riqueza; g) Que sea solidario, } \\
\text { es decir, que se ocupe de los menos } \\
\text { favorecidos; h) otros }\end{array}$ & 139 & 99 & 39 & 91 & 82 & 120 & 66 & 63 \\
\hline $\mathbf{R M}$ & $\begin{array}{l}\text { ¿A través de qué medios preferi- } \\
\text { rías recibir información de un pro- } \\
\text { yecto para decidir si lo apoya?: a) } \\
\text { Una plataforma de internet; b) Co- } \\
\text { rreo electrónico; c) Redes sociales; d) } \\
\text { Por correo tradicional; e) Vía telefó- } \\
\text { nica; f) Otro. }\end{array}$ & 121 & 148 & 73 & 21 & 36 & 13 & & \\
\hline
\end{tabular}




\begin{tabular}{|c|c|c|c|c|c|c|c|c|c|}
\hline Variable & Ítem & $\mathbf{a}$ & b & c & d & e & f & $\mathrm{g}$ & h \\
\hline FC & $\begin{array}{l}\text { ¿Cuál de los siguientes factores } \\
\text { favorecería tu confianza para apo- } \\
\text { yar un proyecto mediante crow- } \\
\text { dfunding?: a) Que en él participen } \\
\text { familiares o amigos; b) Que haya fa- } \\
\text { miliares o amigos de personas que } \\
\text { conozco; c) Que haya personas cono- } \\
\text { cidas, aunque no tengamos vínculos } \\
\text { familiares o de amistad; d) Que tenga } \\
\text { el respaldo de alguna persona o em- } \\
\text { presa reconocidas; e) Que haya sufi- } \\
\text { ciente información disponible sobre } \\
\text { el proyecto en internet; f) Que me ha- } \\
\text { yan enviado información del proyec- } \\
\text { to a través de las redes sociales; g) En } \\
\text { general no me inspiran confianza, ya } \\
\text { que no puedo saber cómo utilizarán } \\
\text { el dinero. }\end{array}$ & 62 & 68 & 86 & 99 & 112 & 41 & 28 & \\
\hline FD & $\begin{array}{l}\text { ¿Cuál de los siguientes factores te } \\
\text { producirían desconfianza al apo- } \\
\text { yar un proyecto mediante crowd- } \\
\text { funding?: a) Que no haya personas } \\
\text { conocidas en el proyecto; b) Que no } \\
\text { tenga página de internet; c) Que no } \\
\text { haya información sobre el proyecto } \\
\text { disponible en internet; d) Que tenga } \\
\text { malas referencias en internet o en las } \\
\text { redes sociales; e) Que pueda estar re- } \\
\text { lacionado con intereses políticos; f) } \\
\text { Que no ofrezca información de resul- } \\
\text { tados a sus inversores o benefactores } \\
\text { (rendición de cuentas); g) Que parez- } \\
\text { ca demasiado utópico o irrealizable. }\end{array}$ & 56 & 74 & 124 & 133 & 124 & 124 & 76 & \\
\hline
\end{tabular}

Nota: En algunas preguntas la sumatoria es mayor a 242 debido a que se permitía más de una respuesta.

Fuente: Elaboración propia.

A continuación, se presentan algunos de los hallazgos organizados conforme a las fases del proceso de traducción.

\subsubsection{Problematización}

El 84\% de los encuestados manifiesta haber tenido algún grado de afectación económica como resultado de la contingencia por la COVID-19, que van desde la pérdida del empleo, el desempleo de algún familiar o conocido, o la reducción de los ingresos familiares.

\subsubsection{Interés}

El 22\% de los menores de 30 años conoce el concepto de crowdfunding o financiamiento colectivo, mientras que en población de 30 años y más, el porcentaje de 
personas que conocen el concepto es de 17,5\%. En menores de 30 años, el 29\% participaría en campañas de crowdfunding si esto permite crear nuevos empleos, y $25 \%$ si ayuda a salvar los ya existentes, mientras que el $26 \%$ prefiere propuestas que generen innovación y el 17\% se enfoca hacia causas altruistas. En mayores de 30 años, el mayor interés se orienta hacia la creación de nuevos empleos (35\%), innovación (22\%), salvar los empleos ya existentes (20\%) y altruismo (16\%) (Figura 3 ).

Los excedentes económicos que pueden ser destinados para el financiamiento colectivo se han reducido durante la contingencia; a pesar de ello el 23\% de los encuestados manifiesta tener excedentes que le permiten ahorrar e invertir.

\section{Figura 3. Aspectos valorados por los inversionistas potenciales en los proyectos a fondear}

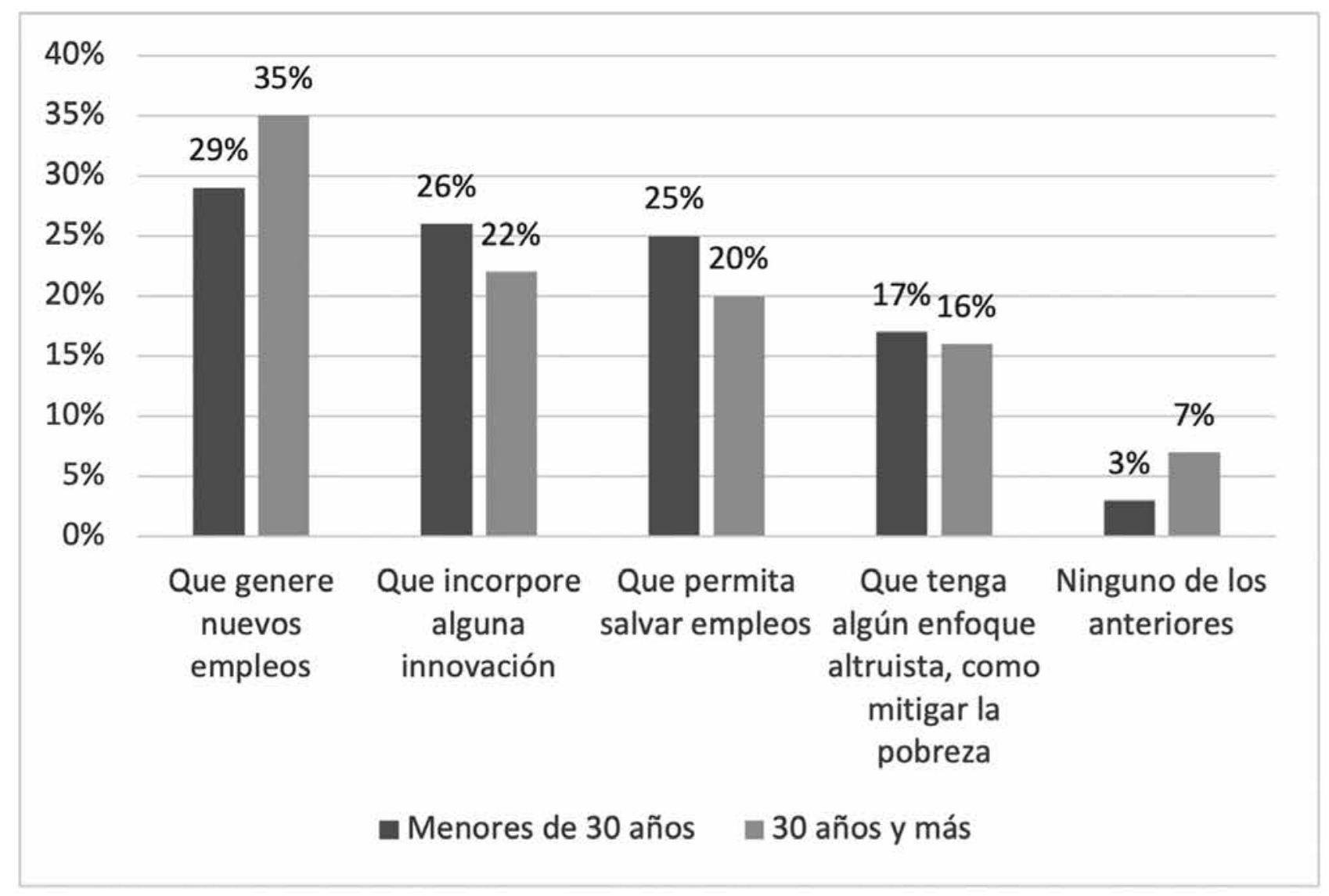

Fuente: Elaboración propia.

En caso de participar en una campaña de crowdfunding, las personas menores de 30 años esperarían recibir a cambio, en primer lugar, información sobre el proyecto y el uso de los recursos (32\%); un segmento de los inversionistas (25\%) esperaría convertirse en socios de los proyectos, y un 11\% esperaría únicamente una recompensa simbólica (Figura 4). 


\section{Figura 4. Expectativas de los inversionistas potenciales al participar en el fondeo de microproyectos}

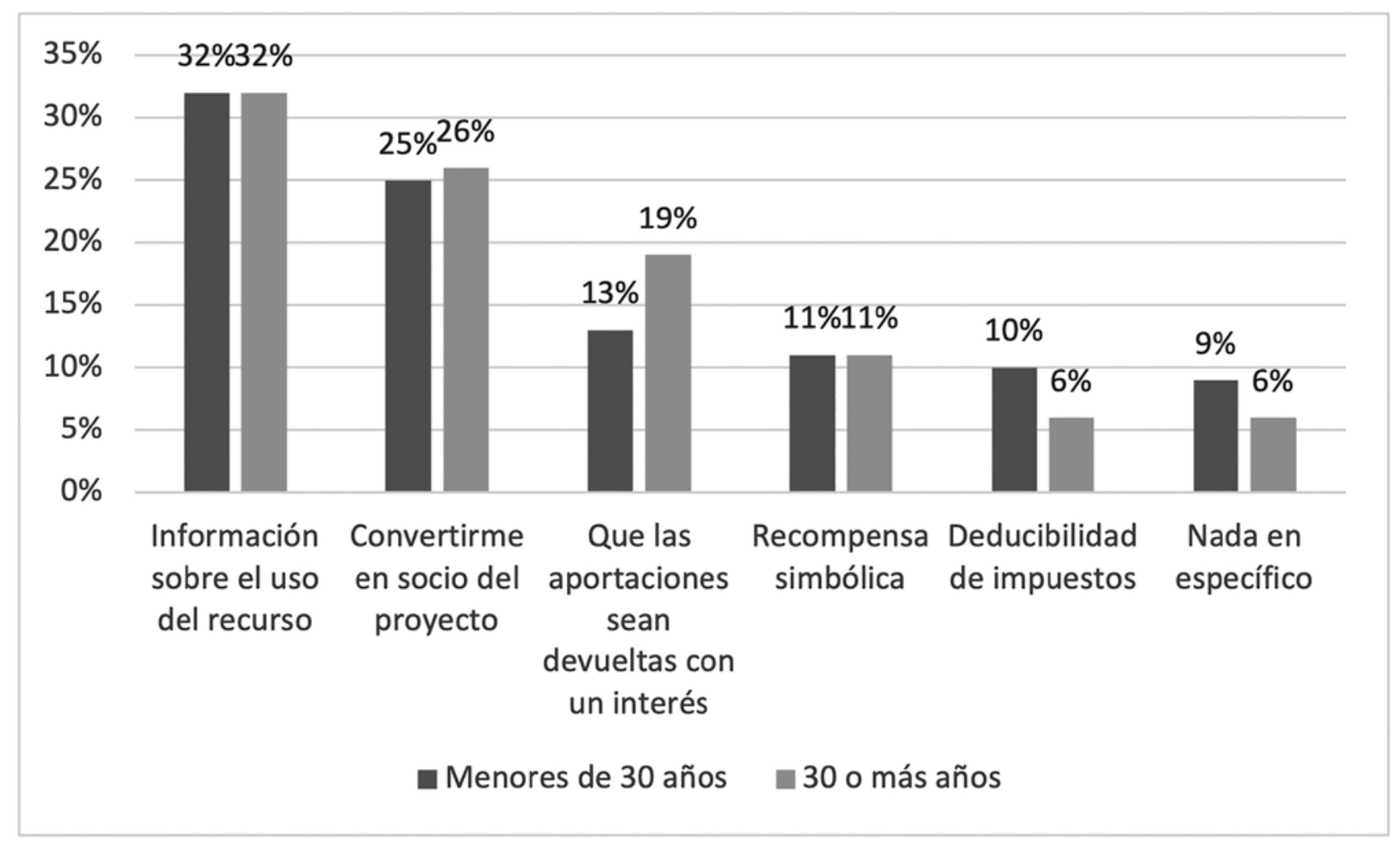

Fuente: Elaboración propia.

\subsubsection{Incorporación}

El 6,5\% de los menores de 30 años ha participado en alguna campaña de donación o financiamiento colectivo, mientras que en mayores de 30 años el nivel de participación es de 5,6\%.

El correo electrónico es mencionado como el medio de contacto predominante para quienes estarían interesados en participar en campañas de CF (45\% en población de 30 o más años y 36\% en menores de 30 años), seguido en importancia por una plataforma en internet (33\% y 32\% respectivamente). Las redes sociales tendrían mayor presencia en menores de 30 años (22\%) que en la población mayor (14\%) (Figura 5).

Los encuestados manifiestan preferencia por apoyar proyectos de su propia localidad (39\%) y de personas conocidas (31\%). La información que requerirían para tomar la decisión de apoyar un proyecto es la descripción general de la empresa, giro y productos (37\%); perfil de los emprendedores (23\%); y la situación fiscal y financiera del proyecto $(22 \%)$.

Los factores que incentivan la confianza en los proyectos, con fines de financiamiento colectivo son, en primer lugar, que en ellos participen familiares, o familiares de personas conocidas (26\%); que haya suficiente información disponible sobre ellos en internet (22\%); y que tenga el respaldo de alguna persona o empresa reconocidas $(20 \%)$. En contraparte, los factores que generan desconfianza son en primer lugar, las malas referencias en internet (18\%) y que no haya información disponible sobre el proyecto en internet (17\%). 


\section{Figura 5. Mediadores tecnológicos preferidos por los inversionistas potenciales}

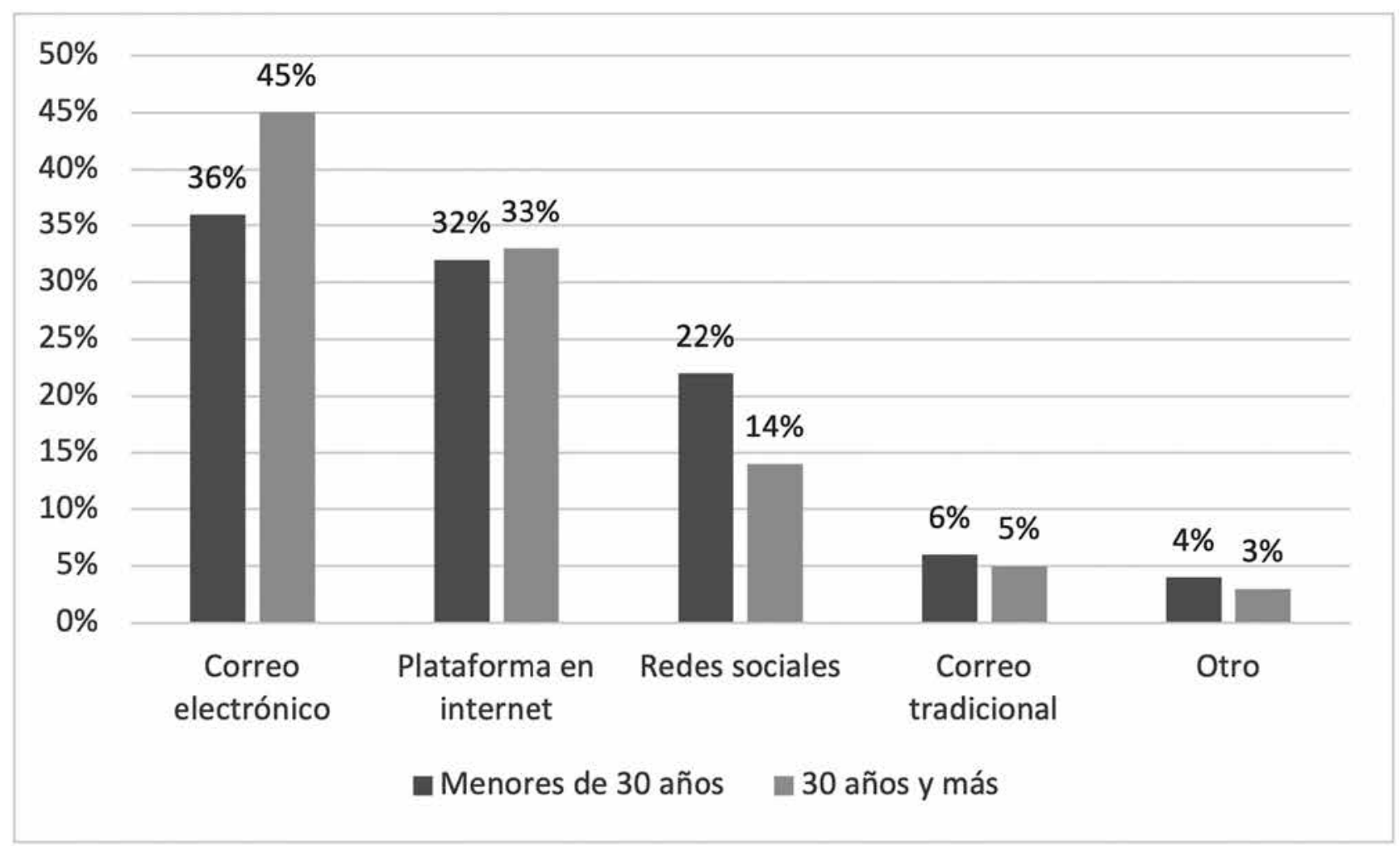

Fuente: Elaboración propia.

\subsubsection{Movilización}

De acuerdo con Gunawong y Gao (2010), la movilización comprende tanto un modelo de implementación, como canales para que los diferentes actores alineen sus intereses y acciones a la ruta propuesta por el actor focal. En México existe un ecosistema de $\mathrm{CF}$, que aglutina a cuatro plataformas de donaciones y recompensas, cinco plataformas de equity, cinco plataformas inmobiliarias, 11 plataformas de préstamos y una plataforma de regalías (Asociación de Plataformas de Fondeo Colectivo-AFICO, 2020); sin embargo, su nivel de penetración es aún incipiente, pues al menos el 78\% de las personas encuestadas desconoce el concepto de financiamiento colectivo. Puede identificarse la existencia de un actor focal (AFICO) y un mecanismo de participación, (ecosistema y plataformas de crowdfunding). Además, hay un proceso de institucionalización de las soluciones propuestas (Ley Fintech).

\subsection{Identificación de asociaciones rastreables}

En la tercera etapa del análisis, se utilizó como herramienta el algoritmo CHAID (Chi-Square Automatic Interaction Detection) con XLSTAT-2016. Se trata de un algoritmo predictivo de segmentación jerárquica que trabaja con una variable dependiente categórica nominal u ordinal, a partir de la cual se crean tantas particiones como variables independientes son consideradas para el modelo. El CHAID utiliza el Chi-cuadrado para estudiar todos los valores de cada variable predictora, lo que ayuda a encontrar qué tan similares o asociadas están las variables y cuál es el predictor más significativo (Sanz \& Ponce de León, 2010). De esta manera, se va formando un árbol de clasificación en el que cada nodo se conforma por categorías similares de la variable seleccio- 
nada. El análisis CHAID permite formular modelos predictivos parecidos al análisis de regresión, así como detectar la interacción entre variables categóricas. Al tratarse de una técnica de segmentación jerárquica, identifica grupos homogéneos de sujetos a partir de los valores que toman las variables criterio en función de la significación estadística de las variables explicativas (valor-p).

Para realizar el análisis, se tomó como variable criterio la Disponibilidad para realizar aportaciones vía CF. Se trata de una variable categórica con tres valores posibles: $0=$ No está dispuesto a realizar aportaciones; 1 = Estaría dispuesto a realizar microaportaciones (menores a 1000 pesos); y 2= estaría dispuesto a realizar aportaciones de capital (mayores a 1000 pesos). En primer lugar, se aplicó el algoritmo de clasificación y regresión a la variable criterio y las variables predictoras correspondientes al perfil del inversionista (edad, género, escolaridad, percepción de cambios en la situación económica asociados a la COVID-19, grado de afectación económica asociada a la COVID-19 y enfoque altruismo vs. provecho personal). Una parte de los resultados se pueden ver en la Figura 6.

\section{Figura 6. Árbol de clasificación para la variable criterio y el perfil del inversionista}

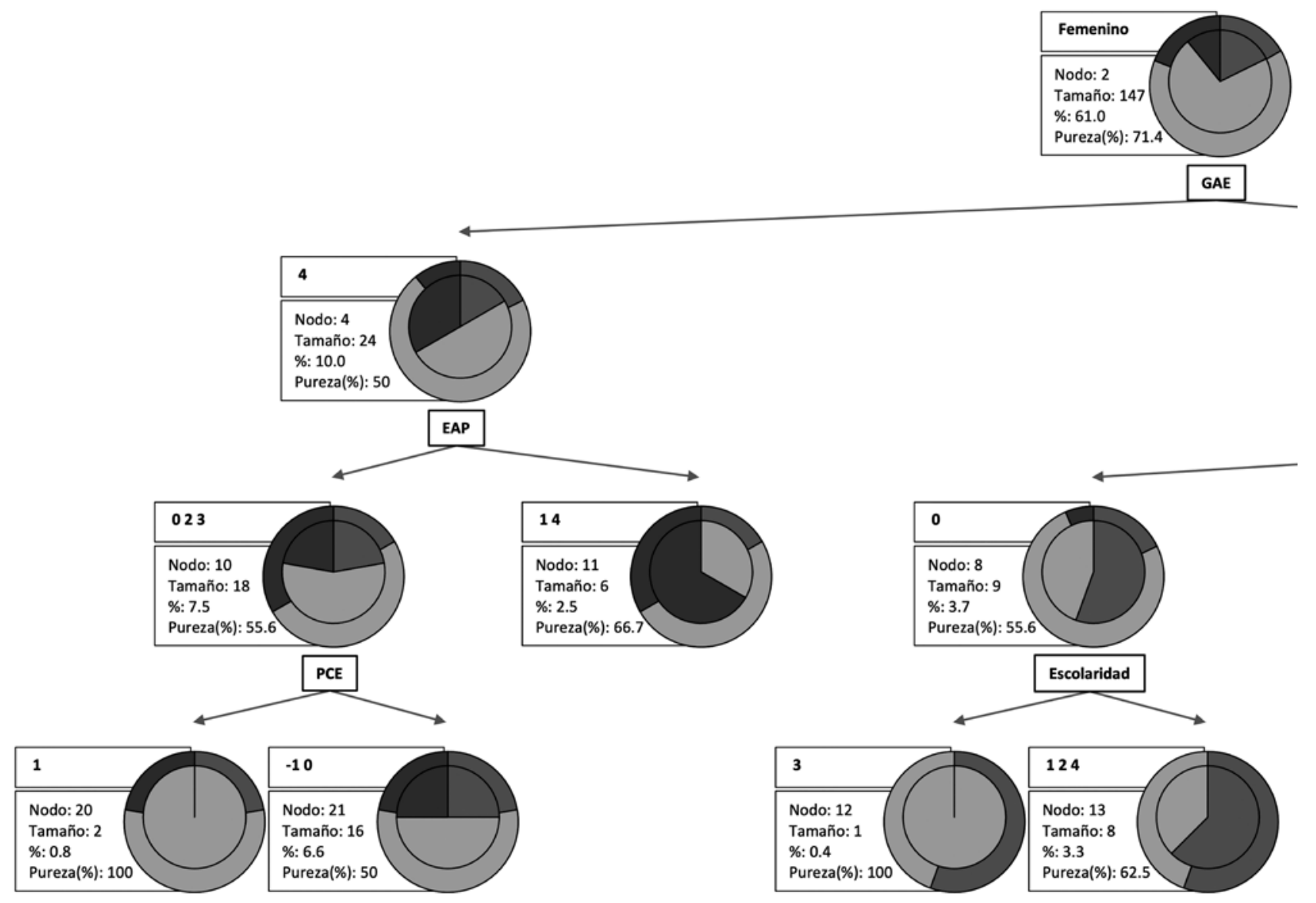

Fuente: Elaboración propia utilizando XLSTAT-2016.

Cada nodo representa un grupo homogéneo con características específicas, cuya capacidad predictiva se da cuando se cumplen condiciones del tipo Si p, entonces q. Por ejemplo, el nodo 20 de la Figura 6, nos muestra un grupo de personas que están dispuestas a realizar microinversiones de capital (la variable criterio tiene valor de 1). Las reglas de este nodo son: 
Si $P C E=1$ (Percibe que la situación económica ha mejorado); EAP = 2,3 (Apoyaría proyectos que generen empleo o que sean creativos e innovadores); GAE=4 (Algún conocido perdió su empleo durante la contingencia) Gen=2 (Femenino) entonces Disponibilidad para Realizar Aportaciones $=1$ en el 100\% de los casos.

Otro ejemplo sería el nodo 11, que perfila a un grupo dispuesto a realizar aportaciones de capital (la variable criterio tiene valor $=2$ ). Las reglas de este nodo son:

Si $E A P=1$ o 4 (Apoyaría proyectos que generen algún beneficio económico y tengan un fin altruista) y $\mathrm{GAE}=4$ (Algún conocido perdió su empleo durante la contingencia) y Gen $=2$ (Femenino) entonces Disponibilidad para Realizar Aportaciones = 2 en el $66,7 \%$ de los casos.

A continuación, se replicó el algoritmo de clasificación para la variable criterio y las variables relacionadas con el perfil de los proyectos de emprendimiento: Tipo de beneficios económicos que ofrece, enfoque local vs. global, factores que hacen atractivo a un proyecto, recursos tecnológicos asociados a la mediación, factores que generan confianza factores que generan desconfianza. De este análisis surgieron 27 nodos y como ejemplo se explican las reglas de los nodos 10 y 23.

Si $R M=2$ (El proyecto se promueve por correo electrónico y una plataforma); $\mathrm{FC}=2,3,4,5$ (En el proyecto participan familiares, amigos o conocidos, hay información sobre el proyecto en internet, los emprendedores envían información vía correo, plataforma o redes sociales); $\mathrm{FA}=1,2$, 3, 4, 6, 7, 8 (El proyecto es contado en forma atractiva, está relacionado con bienes sociales, produce empleos y riqueza, muestra experiencias exitosas, muestra testimonios de personas a las que haya apoyado, y plantea alguna innovación o desarrollo tecnológico) y $\mathrm{LG}=1,2$, 3, (El proyecto involucra empresas de personas conocidas, de mi localidad o de otra localidad en México), entonces Disponibilidad para Realizar Aportaciones $=2$ en el 61,5\% de los casos.

$\boldsymbol{S i} \mathrm{FC}=2$ (Participan en el proyecto familiares o personas conocidas); $\mathrm{RM}=1,2,3$ (Los emprendedores envían información vía correo electrónico, plataforma o redes sociales); $\mathrm{BE}=1,2,3$ (El proyecto ofrece información sobre el uso de los recursos, podría devolver las aportaciones como si fuera un préstamo u ofrece una recompensa simbólica) y $\mathrm{FA}=5,9,10,11$ (El proyecto inspira confianza, propone hacer cosas de alta calidad, es factible, no muy utópico, es solidario) y ELG=1,2,3 (El proyecto involucra empresas de personas conocidas, de mi localidad o de otra localidad en México) entonces Disponibilidad para Realizar Aportaciones = 1 en el 83,3\% de los casos.

Finalmente, se muestra la matriz de confusión (tabla 6) en la que se puede apreciar la exactitud del modelo. El número de aciertos, que en la tabla aparecen en negritas, nos indican que el modelo tiene una capacidad predictiva moderada, pues solo acertó en el $72 \%$ de los casos, por lo que, en una investigación subsecuente, sería conveniente ampliar la muestra y ajustar el cuestionario para incrementar la confiabilidad del algoritmo.

\section{Tabla 6. Matriz de confusión para la muestra de estimación}

\begin{tabular}{|c|c|c|c|c|c|}
\hline $\begin{array}{c}\text { Estimado } \\
\text { Real }\end{array}$ & $\mathbf{0}$ & $\mathbf{1}$ & $\mathbf{2}$ & Total & \% correcto \\
\hline 0 & $\mathbf{1 9}$ & 22 & 0 & 41 & $46,34 \%$ \\
\hline 1 & 3 & $\mathbf{1 4 7}$ & 5 & 155 & $94,84 \%$ \\
\hline 2 & 2 & 34 & $\mathbf{9}$ & 45 & $20,00 \%$ \\
\hline Total & 24 & 203 & 14 & 241 & $72,61 \%$ \\
\hline
\end{tabular}

Fuente: Elaboración propia utilizando XLSTAT-2016. 


\section{Conclusiones y discusión}

La metodología ANT propone construir una narrativa en la que se puede trazar una red parecida a una partitura que vincula actores heterogéneos e inconstantes desde un punto de vista oligóptico, que permite ver poco, pero ver bien (Seijo, 2006). Al seguir la secuencia de problematización- interés- incorporación - movilización, podemos rastrear los movimientos del actor-red, y esto ayuda a comprender la naturaleza de las transacciones que pueden darse entre un inversionista y un emprendedor.

En el entramado del ecosistema inversionista-emprendedor cada acción es motivada por los movimientos de otros participantes, sus expectativas y sus intereses. Esto es a lo que Greimas y Courtès (1982) llaman "actantes", es decir, quien al mismo tiempo actúa y sufre una acción. Tirado et al. (2005), lo definen como cualquier entidad que produzca una relación o adquiera valor de significación, y que se define por su capacidad para producir una acción dentro de una trama.

En respuesta a nuestra primera pregunta de investigación, podemos concluir que sí es posible rastrear las asociaciones entre actores, y que estas ocurren a través de una serie de elementos tangibles e intangibles agrupados en un factor que llamamos "mediación”. El análisis de clasificación y regresión nos permite visualizar la forma en la que los actores de la red se vinculan mediante elementos simbólicos correspondientes a esta variable. Por ejemplo, un inversionista está dispuesto a comprometer sus recursos en un proyecto cuando lo visualiza como confiable y atractivo; esto se da en especial, cuando hay participación de familiares o personas conocidas; es de la misma comunidad que el inversionista; tiene presencia en internet; ofrece información sobre el uso de los recursos; y genera un bien social, como empleos o riqueza, entre otros criterios.

La mediación ha sido ampliamente discutida en el contexto de la ANT y tiene varios significados o dimensiones. Puede entenderse como traducción de metas y como coordinación entre actores y actantes (mediación técnica), pero también como proceso de pliegue espacio-tiempo o caja negra donde "diversos elementos, metas, acciones dadas en otros tiempos y diferentes espacios coexisten comprimidos, plegados, en un único actante" (Correa-Moreira, 2012, p. 69).

El factor mediación también da la pauta para responder a nuestra segunda pregunta de investigación, pues sugiere aspectos que una microempresa o un proyecto de emprendimiento deberían reunir para "producir una acción dentro de la trama". En este sentido, una microempresa o proyecto resultan significativos para los inversionistas y pueden contribuir a su movilización si responden afirmativamente a preguntas clave como: ¿ayuda a resolver alguna problemática que me afecta?; ¿permite que me involucre? y al hacerlo ¿me proporciona una recompensa social o económica?; ¿me inspira un nivel razonable de confianza?; ¿me permite aportar recursos de acuerdo a mis posibilidades?; ¿es fácil acceder a la información del proyecto?; ¿involucra a personas o empresas conocidas?; ¿está visible en internet?; irinde cuentas de los recursos que recibe?; ¿opera en mi localidad?; ¿qué referencias tiene?

Llama la atención cómo la presencia en internet, las referencias positivas y negativas sobre el negocio y la posibilidad de acceder a información sobre el uso de los recursos, son clave para que un inversionista potencial considere que un proyecto de emprendimiento es confiable, lo que nos lleva a concluir que la digitalización es un requisito imprescindible no solo para activar las ventas, sino para acceder al financiamiento a través de CF. Esto es consistente con los hallazgos de Ortega (2020), quien destaca la importancia de diseñar estrategias de marketing digital enfocadas al cliente más que al producto. Además de lo anterior, es relevante la presencia de familiares y conocidos en los proyectos, pues son generadores de confianza, lo que a su vez se relaciona con la confiabilidad de la red de contactos (Gamero \& Ostos, 2020). 
Es importante destacar que, en concordancia con lo advertido por autores como Belleflame et al. (2012), pese a que en el escenario de confinamiento se redujeron los ingresos de la mayor parte de la población, casi una cuarta parte de las personas económicamente activas cuenta aún con excedentes económicos que los convierten en inversionistas potenciales.

Finalmente, la preferencia de los inversionistas potenciales por los proyectos locales denota el efecto positivo de lo que Agrawal et al. (2011) llaman "la geografía del crowdfunding", es decir, la posibilidad de conectar casi sin intermediarios a los inversionistas con los emprendedores.

Con esta información, es posible avanzar en el diseño de una plataforma de financiamiento colectivo que cumpla el rol de mediador y ayude a incrementar el flujo de recursos hacia las microempresas. Más aún, el creciente interés por microproyectos que apoyen en la creación o conservación de empleos en el ámbito local, permite ver que es posible desarrollar redes solidarias de financiamiento, con una estructura descentralizada y con un enfoque no necesariamente lucrativo. Para fines prácticos, este tipo de asociaciones podrían apoyar en el desarrollo de campañas de CF, e inclusive, en el desarrollo de algoritmos que sirvan como base para el diseño de plataformas de microfinanciamiento.

\section{Referencias}

Abbasi, K., Alam, A., Du, M.A., \& Huynh, T.L.D. (2020). FinTech, SME efficiency and national culture: evidence from OECD countries. Technological Forecasting and Social Change, 163, 120454. https://doi.org/10.1016/j.techfore.2020.120454

Agrawal, C., Catalini, C., \& Goldfarb, A. (2011). Offlinerelationships, distance made the internet: the geography of crowdfunding. National Bureau of Economic Research. https://bit.ly/394j9eN

Asociación de Plataformas de Fondeo Colectivo-AFICO (2020). Ecosistema del crowdfunding mexicano. http://bit.ly/36GiE9u

Baltar, F., \& Gorjup, M. T. (2012). Muestreo mixto online: Una aplicación en poblaciones ocultas. Intangible Capital, 8(1), 123-149. http://dx.doi.org/10.3926/ic.294

Bahena, L.E.M., Velázquez, A.R., \& Hernández, A.F. (2017). El crowdfunding en México, una alternativa real de financiamiento e inversión para la mujer emprendedora. UPGTO Management Review, 2(2), 1. http://bit.ly/39XsvIR

Banco de México (Banxico) (2015). Reporte sobre las condiciones de competencia en el otorgamiento de crédito a las pequeñas y medianas empresas (PYME). México. https://bit.ly/2LQ4yLl

Banco Interamericano de Desarrollo (2020). ¿Cómo impactará el COVID-19 al empleo? Posibles escenarios para América Latina y el Caribe. https://bit.ly/3jqInYE

Belleflamme, P., Lambert, T., \& Schwienbacher, A. (2012). Crowdfunding. Tapping the wright crowd. Journal of Business Venturing, 29(5), 585-609. https://doi.org/10.1016/j.jbusvent.2013.07.003

Demirguc-Kunt, A., Klapper, L., Singer, D., \& Van Oudheusden, P. (2015). The Global Findex Database 2014: measuring financial inclusion around the world. https://bit.ly/3p2R0dS

Echeverría, J., \& González, M. I. (2009). Actor-network theory and the thesis of technoscience. Arbor-ciencia Pensamiento y Cultura, 185(738), 705-720. https://doi.org/ 10.3989/arbor.2009.738n1047

Gamero, H., \& Ostos, J. (2020). Revisión sistemática de literatura sobre factores clave en la identificación de oportunidades de negocio. Retos. Revista de Ciencias de la Administración y Economía, 10(20), 307-327. https://doi.org/10.17163/ret.n20.2020.07

García, M.A. (2017). Encuentro en el laboratorio: la teoría del actor-red y la escena musical pilagá. Indiana, 34(1), 309-329. https://doi.org/10.18441/ind.v34i1.309-329 
García de León, S., \& Garibay, R. (2016). Financiamiento alternativo: crowdfunding para pequeños y medianos proyectos empresariales e iniciativas sociales. Hospitalidad ESDAI, (29), 35-52. https://bit.ly/2M5Y4ru

González, J., Valdés, F., \& Saavedra, M. (2021). Factores de éxito en el financiamiento para Pymes a través del crowdfunding en México. Revista Mexicana de Economía y Finanzas Nueva Época REMEF, 16(2) 1-23. https://doi.org/10.21919/remef.v16i2.471

González, K.M. (2018). An Overview of Fintech in the Mexican Entrepreneurial Ecosystem. Network Intelligence Studies, 6(12), 141-148. http://bit.ly/39T2tpM

Greimas, A., \& Courtès, J. (1982). Semiotics and Language. An Analytical Dictionary. Indiana University Press.

Gunawong, P., \& Gao, P. (2010). Challenges of eGovernment in Developing Countries: actor-network analysis of Thailand's smart ID card project. Proceedings of the 4th ACM/IEEE International Conference on Information and Communication Technologies and Development (pp. 1-9). ACM Press. https://doi.org/10.1145/2369220.2369235

Heckathorn, D. (1997). Respondent-driven sampling: A new approach to the study of hidden populations. Social Problems, 44(2), 174-199. http://dx.doi.org/10.1525/sp.1997.44.2.03x0221m

INEGI, C.E. (2019). Censos económicos 2019. Resultados oportunos. https://bit.ly/3ixpgLZ

INEGI, EDN (2020). Estudio sobre la demografía de los negocios 2020. Primer conjunto de resultados. https://bit.ly/3sKWbRK

Iyamu, T., Nehemia-Maletzky, M., \& Shaanika, I. (2018). The use of activity theory and actor network theory as lenses to underpin information systems studies. J. Syst. Inf. Tehonology, 20(2). http://dx.doi.org/10.1108/JSIT-10-2017-0098

Jarrahi, M.H., \& Sawyer, S. (2019). Networks of innovation: the sociotechnical assemblage of tabletop computing. Research Policy, X(1), 100001. https://doi.org/10.1016/j.repolx.2018.100001

Latour, B. (2008). Reensamblar lo social. Una introducción a la teoría del actor-red. Manantial.

Law, J. (2009). Actor network theory and material semiotics. Social theory, 141. https://bit.ly/2YMaMPq

León, E.L., \& Saavedra, M.L. (2018). Fuentes de financiamiento para las MIPYME en México. Cambio climático. Caso de estudio: evaluación del Programa del Cambio Climático del Estado de Chihuahua, 113. https://bit.ly/3qQ5LRV

Marpsata, M., \& Razafindratsimab, N. (2010). Survey methods for hard-to-reach populations: introduction to the special issue. Methodological Innovations Online, 5(2), 3-16. https://doi.org/10.4256/mio.2010.0014

Correa-Moreira, G. (2012). El concepto de mediación técnica en Bruno Latour Una aproximación a la teoría del actor-red. Psicología, Conocimiento y Sociedad, 2(1), 56-81. https://bit.ly/3b6rVKl

Nelson, A. (2020). COVID-19: Capitalist and postcapitalist perspectives. Human Geography, 13(3), 305-309. https://doi.org/10.1177/1942778620937122

Ortega, M. (2020). Efectos del Covid-19 en el comportamiento del consumidor: Caso Ecuador. Retos. Revista de Ciencias de la Administración y Economía, 10(20), 233-247 https://doi.org/10.17163/ret.n20.2020.03

Oxford Analytica (2019). "Mexico fintech sector to boom as the economy struggles". Expert Briefings. https://doi.org/10.1108/OXAN-DB245174

Ruiz, R. (1999). Las traducciones dinámicas de las series temáticas: propuesta de una nueva clasificación. En La representación y la organización del conocimiento en sus distintas perspectivas. Su influencia en la recuperación de la información. Actas del IV Congreso ISKO-España EOCONSID 99. 22-24 de abril de 1999, Granada (pp. 211-216). Universidad de Granada. https://bit.ly/3sEiTKK

Schwienbacher, A., \& Larralde, B. (2010). Crowdfunding of small entrepreneurial ventures. Handbook of entrepreneurial finance, Oxford University Press, Forthcoming.

http://dx.doi.org/10.2139/ssrn.1699183

Salido, N., Rey, M., Álvarez, L.I., \& Vázquez, R. (2019). Determinants of success of donation based crowdfunding through digital platforms: The influence of offline factors. CIRIEC - España, Revista de Economía Pública, Social y Cooperativa, (95), 119-141. https://doi:10.7203/CIRIEC-E.95.13001 
Salvador, D. (2018). La inversión privada en el Crowdfunding lucrativo: crecimiento, nuevas modalidades y constitución de Plataformas de financiación participativa. Revista Española de Capital Riesgo, 2, 5-26. http://bit.ly/3oYfN2C

Sánchez, M., Zalba, P., \& Zoppis, J. (2020). Evolution of Fintechs in Argentina. Dimensión Empresarial, 18(4). https://doi.org/10.15665/dem.v18i4.2322

Sanz, E., \& Ponce de León, A. (2010). Claves en la aplicación del algoritmo Chaid: un estudio del ocio físico deportivo universitario. Revista de Psicología del Deporte, 19(2), 319-333. https://bit.ly/3aE9XgQ

Seijo, G. (2006). Reseña de "Reassembling the social. An introduction to Actor-Network-Theory" de Bruno Latour. Redes, 12(24), 151-161. https://bit.ly/3sHnSuW

Secretaría de Hacienda y Crédito Público, SHCP (2018). Ley para regular las Instituciones de Tecnología Financiera. https://bit.ly/2Y0Itfs

Tirado, F., López, D., Callén, B., \& Domènech, M. (2008). La producción de fiabilidad en entornos altamente tecnificados. Apuntes etnográficos sobre un servicio de teleasistencia domiciliaria. Papeles del CEIC, 2(38). Universidad del País Vasco. https://bit.ly/3c8BSYy

Zedeli, K. (2019). The role of Fintech in promoting financial inclusion in developing countries: the case of Mexico. (Master Thesis). Berlin School of Economics and Law. http://bit.ly/2NqFDP7 